\title{
Rainbow spiral emission from optical fibers
}

\author{
F. Mangini ${ }^{1}$, M. Ferraro ${ }^{2}$, M. Zitelli ${ }^{2}$, V. Kalashnikov ${ }^{2}$, A. Niang ${ }^{1}$, T. Mansuryan ${ }^{3}$, \\ F. Frezza ${ }^{2}$, A. Tonello ${ }^{3}$, V. Couderc ${ }^{3}$, A.B. Aceves ${ }^{4}$, S. Wabnitz ${ }^{2,5}$ \\ ${ }^{1}$ Department of Information Engineering (DII), University of Brescia, Via Branze 38, 25123 Brescia, Italy \\ ${ }^{2}$ Department of Information Engineering, Electronics and Telecommunications (DIET), Sapienza University of \\ Rome, Via Eudossiana 18, 00184 Rome, Italy \\ ${ }^{3}$ Université de Limoges, XLIM, UMR CNRS 7252, 123 Avenue A. Thomas, 87060 Limoges, France \\ ${ }^{4}$ Department of Mathematics, Southern Methodist University, 3100 Dyer St, Dallas, TX 75205, USA \\ ${ }^{5}$ Novosibirsk State University, Pirogova 1, Novosibirsk 630090, Russia
}

\begin{abstract}
We observed the generation of a spiral-shaped intensity beams from optical fibers under suitable coupling conditions. In nonlinear regime, we observed rainbow-spiral emission thanks to the generation of supercontinuum in the fiber.@ 2021 The Author(s)
\end{abstract}

A common method to obtain spiral-shaped beams involves interfering a plane wave with a so-called longitudinal orbital angular momentum (LOAM) beam. Studies of LOAM beams, exhibiting phase singularities and helical wavefronts, date back to the early nineties [1]. For these beams, the electric field is proportional to $e^{i \ell \varphi}$, where $\varphi$ is the azimuthal angle, and $\ell$ is the number of wavefront screwing per wavelength.

In addition to LOAM beams, studies of the so-called transverse orbital angular momentum (TOAM) beams have emerged in recent years [2]. TOAM beams, which are characterized for having parallel propagation and average Poynting vectors, may exhibit an helical trajectory, and generate spiral beams. In this work, we demonstrate a simple method for generating spiral emission via TOAM beams, based on the controlled coupling of a Gaussian laser beam with cladding modes of commercial, singlemode or multimode optical fibers. Our approach does not require any modification to the fiber structure, since the TOAM beam is generated by geometrical constraints. Specifically, TOAM beams are obtained by focusing the laser beam inside the cladding, at the input facet of the fiber, with proper incidence angles $\vartheta\left(<2^{\circ}\right)$ and $\varphi$. As a result, the wave vector projection on the fiber input facet of the incident field, $k_{t}$, is tangent to the core-cladding interface (see Fig.1a).

In this way, the azimuthal component of the wave vector forces light to travel on a helical path around the fiber core, which spontaneously provides the phase profile, which is necessary to generate spiral emission in the intensity of the far-field at the fiber output, as depicted in Fig.1a. Remarkably, the helical path of the beam can be directly visualized by using high peak power ultrashort optical pulses, thanks to the associated luminescence signal from fiber defects and impurities [3]. In Fig.1b we show the case of a graded-index (GRIN) fiber, whose germanium doping produces blue luminescence. Note that, if the beam is focused into the core, spiral emission cannot be produced, and luminescence generates an array of bright spots, owing to spatial self-imaging [4].
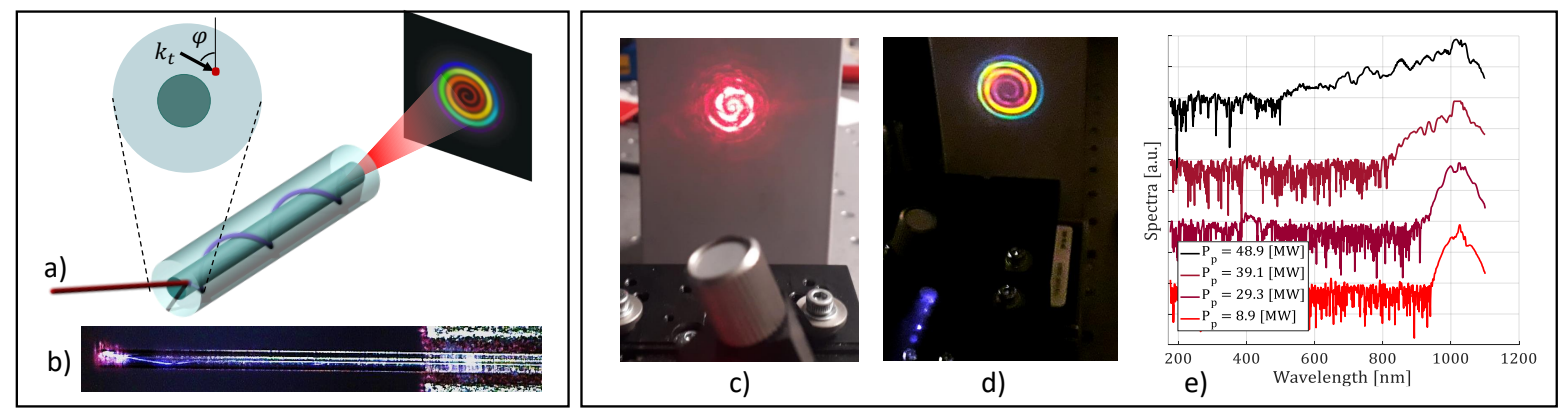

Fig. 1. a) Schematic of spiral emission generation; b) Luminescence signal revealing the helical path of the beam; c) True-colour picture of far-field emission from a standard monomode fiber (SMF), coupled with a $3 \mathrm{~mW}$ He-Ne laser; d) True-colour picture of far-field from a 50/125 GRIN MMF, coupled with femtosecond laser, with input peak power of 48.9 MW e) Output spectra vs. input peak power. In all experiments, the input beam diameter is $10 \mu \mathrm{m}$ at $1 / e^{2}$ of peak intensity.

Spiral emission generation via the excitation of cladding modes is a purely linear propagation effect. The output spiral shaped far-field obtained with a CW He-Ne laser and a SMF is shown in Fig.1c. In the nonlinear regime, 
the Kerr effect leads to spectacular spiral emission shaping, associated with supercontinuum (SC) generation. Specifically, with proper coupling conditions the generated wavelengths spontaneously separate in space, leading to spiral-shaped rainbow emission, owing to self-phase modulation in the radial direction $[5,6]$. In Fig. 1d, we show a true color picture obtained with ultrashort pulses at $1030 \mathrm{~nm}$. In Fig. 1e, we show the corresponding spectrum as function of input peak power: a broadband and high-brightness SC covers the whole visible spectral range.

a)

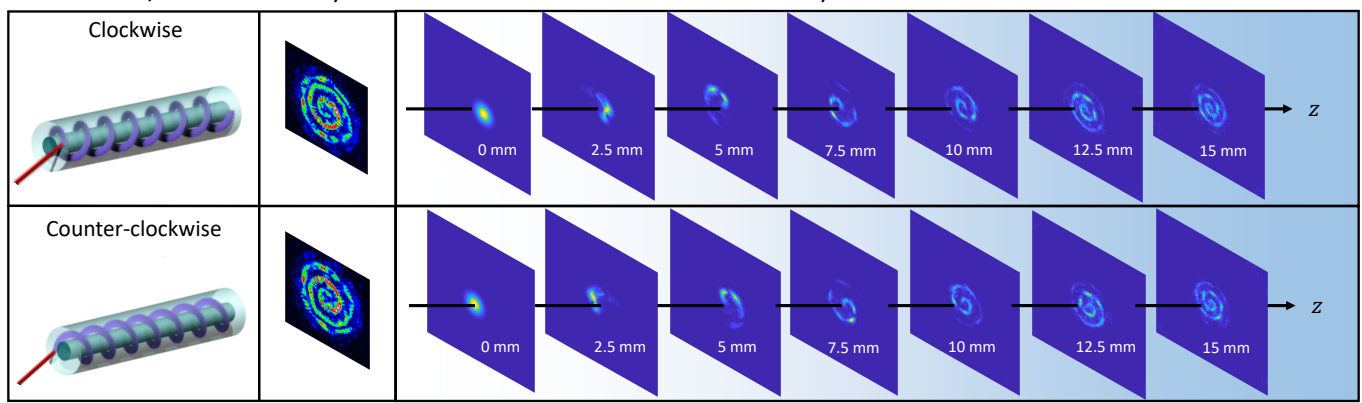

Fig. 2. a) Sketch of clockwise and anticlockwise skew rays; b) Evolution of the far-field intensity, numerically calculated from the Fourier transform of the beam near-field. c) Corresponding experimental far-field intensity measured by a CCD camera. Results obtained for a 50/125 GRIN fibers with $\lambda=1030 \mathrm{~nm}$ for $3 \mathrm{MW}$ input peak power. The beam was injected with $\vartheta=1.5^{\circ}$ and $\varphi=45^{\circ}$.

Helical beam generation and spiral emission are well reproduced by numerical simulations. We used the same model of ref. [7]. In Fig. 2 we compare numerics and experiments as the incidence point of the input beam is varied. As shown in Fig.2a, both clockwise and anticlockwise skew rays can be obtained, by hitting diametrically opposite points in the cladding, while keeping the input tilt angle unchanged. As a result, spiral emission with opposite chiralities is obtained (see the spiral formation process in Fig.2b). Simulations also show that spiral beams generation vanishes beyond a few centimeters of propagation, because of the leaky nature of cladding modes, and the coupling with core modes.

In conclusion, we demonstrated a new approach for the generation of spiral emission in the far-field by using commercially available optical fibers. By exploiting the nonlinear Kerr effect, we observed rainbow-like SC spiral emission. Our results may provide a useful means for the practical generation of spiral beams, that could find applications in several fields, ranging from optical tweezers to data storage, super-resolution, and nanoscale microscopy.

We acknowledge the financial support from the European Research Council (grant No. 740355, STEMS), the Marie Skłodowska-Curie Grant No. 713694 (MULTIPLY), and the Russian Ministry of Science and Education Grant No. 14.Y26.31.0017. French ANR with the "TRAFIC project: ANR-18-CE080016-01"; the CILAS Company (ArianeGroup) with the shared X-LAS laboratory; the "Région Nouvelle Aquitaine" with the projects F2MH and Nematum; the National Research Agency with the reference ANR-10-LABX-0074-01 Sigma-LIM.

\section{References}

1. L. Allen, M. W. Beijersbergen, R. Spreeuw, and J. Woerdman, "Orbital angular momentum of light and the transformation of Laguerre-Gaussian laser modes," Phys. Rev. A 45, 8185 (1992).

2. K. Y. Bliokh and F. Nori, "Transverse and longitudinal angular momenta of light," Phys. Reports 592, 1-38 (2015).

3. F. Mangini, M. Ferraro, M. Zitelli, A. Niang, A. Tonello, V. Couderc, and S. Wabnitz, "Multiphotonabsorption-excited up-conversion luminescence in optical fibers," Phys. Rev. Appl. 14, 054063 (2020).

4. T. Hansson, A. Tonello, T. Mansuryan, F. Mangini, M. Zitelli, M. Ferraro, A. Niang, R. Crescenzi, S. Wabnitz, and V. Couderc, "Nonlinear beam self-imaging and self-focusing dynamics in a GRIN multimode optical fiber: theory and experiments," Opt. Express 28, 24005-24021 (2020).

5. A. Couairon and A. Mysyrowicz, "Femtosecond filamentation in transparent media," Phys. Reports 441, 47-189 (2007).

6. E. Smetanina, A. Dormidonov, and V. Kompanets, "Supercontinuum conical emission accompanying filamentation of a femtosecond laser pulse in fused quartz," J. Opt. Technol. 77, 463-464 (2010).

7. M. Zitelli, F. Mangini, M. Ferraro, A. Niang, D. Kharenko, and S. Wabnitz, "High-energy soliton fission dynamics in multimode GRIN fiber,” Opt. Express 28, 20473-20488 (2020). 